\title{
Combining Fuzzy Logic and Dempster-Shafer Theory
}

\author{
Andino Maseleno*, Md. Mahmud Hasan, Norjaidi Tuah \\ STMIK Pringsewu, Pringsewu, Lampung, Indonesia \\ Faculty of Information Technology, Kazakh British Technical University, Kazakhstan \\ Computer Science Program, Universiti Brunei Darussalam, Negara Brunei Darussalam \\ ${ }^{*}$ Corresponding author, e-mail: andinomaseleno@mail.ru
}

\begin{abstract}
This research aims to combine the mathematical theory of evidence with the rule based logics to refine the predictable output. Integrating Fuzzy Logic and Dempster-Shafer theory is calculated from the similarity of Fuzzy membership function. The novelty aspect of this work is that basic probability assignment is proposed based on the similarity measure between membership function. The similarity between Fuzzy membership function is calculated to get a basic probability assignment. The DempsterShafer mathematical theory of evidence has attracted considerable attention as a promising method of dealing with some of the basic problems arising in combination of evidence and data fusion. DempsterShafer theory provides the ability to deal with ignorance and missing information. The foundation of Fuzzy logic is natural language which can help to make full use of expert information.
\end{abstract}

Keywords: fuzzy logic, Dempster-Shafer theory, membership function, basic probability assignment

Copyright $@ 2015$ Institute of Advanced Engineering and Science. All rights reserved.

\section{Introduction}

Large amount of literature is available on Fuzzy Logic and its applications. Fuzzy Logic can handle problems with imprecise data and give more accurate results. Professor L.A. Zadeh introduced the concept of Fuzzy Logic [1]. Several researchers have investigated the relationship between Fuzzy sets and Dempster-Shafer mathematical theory of evidence and suggested different ways of integrating them. Integration within symbolic, rule-based models have been used for control and classification purposes [2, 3]. Yager and Filev attempted to present a Fuzzy inference system based on Fuzzy Dempster-Shafer mathematical theory of evidence which integrated the probabilistic information in the output [4]. In their works, the consequent is shaped as a Dempster-Shafer belief structure, where each focal element has the same membership function. Binaghi et al. [3] proposed a structure for classification tasks similar to that of [4], where the focal element is a set representing the class label. Dymova et al.[5] proposed a critical analysis of conventional operations on intuitionistic Fuzzy values and their applicability to the solution of multiple criteria decision making problems in the intuitionistic Fuzzy setting. Ghasemi, et al., [6] studied the main characteristic of the proposed method where is that the rules of Fuzzy inference system are considered as evidences in which the firing level of each rule and Fuzzy Naive Bayes method are employed for calculating the basic probability assignment of focal element. In the Naive Bayes classifier, all variables are assumed to be nominal variables, which mean that each variable has a finite number of values and also assumes independence of features. However, in large databases, the variables often take continuous values or have a large number of numerical values.

Walijewski, et al., [7] concentrated on the role of Fuzzy operators, and on the problem of discretization of continuous attributes. Dutta et al. [9] studied Dempster-Shafer theory of evidence by considering focal elements as triangular Fuzzy number. The authors have devised a method for obtaining belief and plausibility measure from basic probability assignments assigned to Fuzzy focal elements. Boudraa, et al., [8] estimated basic probability assignments using Fuzzy membership functions. Binaghi, et al., [3] presented a supervised classification model integrating Fuzzy reasoning and Dempster-Shafer propagation of evidence has been built on top of connectionist techniques to address classification tasks in which vagueness and ambiguity coexist. The approach is the integration within a Neuro-Fuzzy system of knowledge structures and inferences for evidential reasoning based on Dempster-Shafer theory. The 
common weakness of neural network, however, is a problem of determination of the optimal size of a network configuration, as this has a significant impact on the effectiveness of its performance. The Dempster-Shafer theory originated from the concept of lower and upper probability induced by a multivalued mapping by Dempster [10,11]. Following this work his student Glenn Shafer [12] further extended the theory in his book "A Mathematical Theory of Evidence", a more thorough explanation of belief functions. Lyu et al. [13] studied DempsterShafer theory for the reasoning with imprecise context. Yao, et al., [14] used Dempster-Shafer theory for the multi-attribute decision making problems with incomplete information by identify all possible focal elements from the incomplete decision matrix, and then calculate the basic probability assignment of each focal element and the belief function of each decision alternative. $\mathrm{Yu}$, et al., [15] used Dempster-Shafer Theory as an applied approach to scenario forecasting based on imprecise probability. Uphoff, et al., [16] studied application of Dempster-Shafer theory to task mapping under epistemic uncertainty. Dempster-Shafer mathematical theory of evidence implies a type of uncertainty associated with conditions of ambiguity through the data by dealing with ignorance and missing information. This characteristic is due to using a combination of evidence weight from different sources to obtain a new evidence weight.

\section{Dempster-Shafer Mathematical Theory of Evidence}

It is difficult to avoid uncertainty when attempting to make models of the real world. Uncertainty is inherent to natural phenomena, and it is impossible to create a perfect representation of reality. Classic mathematics deals with ideal worlds where perfect geometric figures exist and can verify extraordinary conditions. The formalisation of Fuzzy sets started in the 1960s with the works of Zadeh [1] in Fuzzy sets and Dempster [11] in belief functions. Belief functions offer a non Bayesian method for quantifying subjective evaluations by using probability. In the 1970s, it was further developed by Shafer, whose book Mathematical Theory of Evidence [12] remains a classic in belief functions, or the so-called Theory of Evidence. This theory has been also called the Dempster-Shafer Mathematical Theory of Evidence. In the 1980s, the scientific community working with Artificial Intelligence got involved in using the theory of evidence in applications. The Dempster-Shafer theory or the theory of belief functions is a mathematical theory of evidence which can be interpreted as a generalization of probability theory in which the elements of the sample space to which nonzero probability mass is attributed are not single points but sets. The sets that get nonzero mass are called focal elements. The sum of these probability masses is one, however, the basic difference between Dempster-Shafer mathematical theory of evidence and traditional probability theory is that the focal elements of a Dempster-Shafer structure may overlap one another. The Dempster-Shafer mathematical theory of evidence also provides methods to represent and combine weights of evidence.

\subsection{Representation of Evidence}

The Dempster-Shafer theory assumes that there is a fixed set of mutually exclusive and exhaustive elements called hypotheses or propositions and symbolized by the Greek letter $\Theta$, represented as $\Theta=\{h 1, h 2, \ldots, h n\}$, where hi is called a hypothesis or proposition. A hypothesis can be any subset of the frame, in example, to singletons in the frame or to combinations of elements in the frame. $\Theta$ is also called frame of discernment. A basic probability assignment (bpa) is represented by a mass function $m: 2^{\Theta} \rightarrow[0,1]$. Where $2^{\ominus}$ is the power set of $\Theta$.

The basic probability assignment is a primitive of evidence theory. Generally speaking, the term basic probability assignment does not refer to probability in the classical sense. The bpa, represented by $m$, defines a mapping of the power set to the interval between 0 and 1 , where the bpa of the null set is 0 and the bpas of all the subsets of the power set is 1 . In Fuzzy Logic, two valued logic often considers 0 to be false and 1 to be true. Fuzzy Logic deals with truth values between 0 and 1 , and these values are considered as the intensity or degrees of truth.

The value of the bpa for a given set $\mathrm{A}$ (represented as $m(A) ; A \in 2^{\Theta}$ ), expresses the proportion of all relevant and available evidence that supports the claim that a particular element of $\Theta$ (the universal set) belongs to the set $A$ but to no particular subset of $A$. The value of $m(A)$ pertains only to the set $A$ and makes no additional claims about any subsets of $A$. Any further evidence on the subsets of $\mathrm{A}$ would be represented by another bpa, in example $B \subset A, \mathrm{~m}(\mathrm{~B})$ 
would the bpa for the subset B. Formally, this description of $m$ can be represented with the following two equations 1 and 2:

$$
\begin{aligned}
& m(\emptyset)=0 \\
& \sum_{A \in 2^{\Theta}} m(A)=1
\end{aligned}
$$

From the primitive of evidence theory or mass function, the upper and lower bounds of an interval can be defined. This interval contains the precise probability of a set of interest and is bounded by two non additive continuous measures called Belief function and Plausibility function. Evidence theory uses two measures of uncertainty, belief function and plausibility function, expressed as $\mathrm{Bel}()$ and $\mathrm{Pls}()$ respectively. Given a basic probability assignment $m$, the corresponding belief function measure and plausibility function measure are determined for all sets $A \in 2^{\ominus}$ and $B \in 2^{\ominus}$ by equations 3 and 4 :

$$
\begin{aligned}
& \operatorname{Bel}(A)=\sum_{B \subseteq A} m(B) \\
& \operatorname{Pls}(A)=\sum_{B \cap A \neq \emptyset} m(B)
\end{aligned}
$$

The support function or belief, Bel, is the total belief of a set and all its subsets. The lower bound Belief for a set $A$ is defined as the sum of all the basic probability assignments of the proper subsets $(\mathrm{B})$ of the set of interest $(\mathrm{A})(A \subset B)$. The plausibility function of a proposition, $\mathrm{Pls}$, is the sum of the masses of all propositions in which it is wholly or partially contained. The plausibility function is defined as the degree to which the evidence fails to refute $A$. These two functions, which have been sometimes referred to as lower and upper probability functions, have the following properties are given by equations 5 and 6 :

$$
\begin{aligned}
& \operatorname{Bel}(A) \leq \operatorname{Pls}(A) \\
& \operatorname{Pls}(A)=1-\operatorname{Bel}(\bar{A})
\end{aligned}
$$

Where $\bar{A}$ is the complementary hypothesis of $A, A \cup \bar{A}=\Theta$ and $A \cap \bar{A}=\emptyset$. The plausibility PIs (A) is defined as the degree to which the evidence fails to refute $A$. This term is given by the equation 7:

$$
P l s(A)=1-\operatorname{Bel}(\bar{A})=1-\sum_{B \subseteq \bar{A}} m(B)
$$

Due to a lack of information, it is more reasonable to present bounds for the result of uncertainty quantification, as opposed to a single value of probability. The total degree of belief in a given proposition $\mathrm{A}$ is expressed within an interval $[\operatorname{Bel}(\mathrm{A}) ; \operatorname{Pls}(\mathrm{A})]$, which lies in the unit interval $[0,1]$.

Dealing with uncertainty is a fundamental issue in the study of man-made computational devices and systems which can be made to act in a manner which human would be inclined to call intelligent. Dempster-Shafer mathematical theory of evidence is an important tool of uncertainty modelling when both uncertainty origins from human's lack of knowledge of the physical world and uncertainty derives from the natural variability of the physical world are present in the problem under consideration.

In decision making processes with human's lack of knowledge of the physical world and lack of the ability of measuring and modelling the physical world, the Fuzzy Logic and 
Dempster-Shafer mathematical theory of evidence have gained prominence as the methods of choice over traditional probabilistic methods. The fundamental and important object of the mathematical theory of evidence is the primitive function called a basic probability assignment. In the absence of empirical data, experts in related fields provide necessary information. However how to obtain basic probability assignment is still an open issue. The membership function of a Fuzzy set is a generalization of the indicator function in classical sets. In Fuzzy Logic, it represents the degree of truth as an extension of valuation. Fuzzy Logic is a logic operation method based on many-valued logic rather than binary logic or two-valued logic.

Dempster-Shafer mathematical theory of evidence, a probabilistic reasoning technique, is designed to deal with uncertainty and incompleteness of available information. DempsterShafer mathematical theory of evidence allows one to combine evidence from different sources and arrive at a degree of belief which is represented by a belief function that takes into account all the available evidence. The degree of belief is expecting a truth value which is the relation between Fuzzy Logic and Dempster-Shafer mathematical theory of evidence.

\subsection{Evidence Combination}

Dempster-Shafer theory provides a method to combine the previous measures of evidence of different sources. This rule assumes that these sources are independent. The combination: $m=m 1 \oplus m 2$, also called orthogonal sum, is defined according to the Dempster's rule of combination [12]. It can be applied repetitively when the sources are more than two. After the combination, a decision can be made among the different hypotheses according to the decision rule chosen.

To use Dempster-Shafer mathematical theory of evidence, there must be the feasible measures to determine basic probability assignment. The Fuzzy theory also requires basic probability assignment. Basic probability assignment which is called the primitive function is the fundamental and important object of the mathematical theory of evidence. The membership function of a Fuzzy set is a generalization of the indicator function in classical sets. In Fuzzy Logic, it represents the degree of truth as an extension of valuation. Fuzzy Logic is a logic operation method based on many-valued logic rather than binary logic or two-valued logic. Twovalued logic often considers 0 to be false and 1 to be true. Fuzzy Logic deals with truth values between 0 and 1 , and these values are considered as the intensity or degrees of truth. Dempster-Shafer mathematical theory of evidence, a probabilistic reasoning technique, is designed to deal with uncertainty and incompleteness of available information. Dempster-Shafer mathematical theory of evidence allows one to combine evidence from different sources and arrive at a degree of belief which is represented by a belief function that takes into account all the available evidence. The Degree of belief is expecting a truth value which is the relation between Fuzzy Logic and Dempster-Shafer mathematical theory of evidence.

\section{Fuzzy Logic}

The original motivation for Fuzzy Logic is to provide the basis for reasoning under nonbinary information. The ensuing reasoning system often this is referred to as approximate reasoning or Fuzzy reasoning. However, this should not be taken to imply that the resulting system is any less exact than that afforded by crisp logic. Indeed, Fuzzy reasoning might be considered more exact precisely because it does not assume a binary universe. The basis for formal reasoning is an inference procedure, itself based upon an appropriate model for 'if-then' rules, or modus ponens. The general goal is to infer the degree of truth associated with a proposition, $\mathrm{B}$, from the implication, $\mathrm{A}$, or $A \rightarrow B$. Consider, ' $A$ ' denotes "sharp corner" and 'B' "approach slowly" than the implication can naturally express by:

premise 1 (fact): $x$ is A; premise 2 (fact): IF $x$ is A THEN $y$ is $B$; consequence (conclusion): $\mathrm{y}$ is $\mathrm{B}$.

Or

premise 1 (fact): $x$ is $A^{\prime}$; premise 2 (fact): IF $x$ is A THEN $y$ is $B$; consequence (conclusion): $y$ is $\mathrm{B}^{\prime}$.

Fuzzy reasoning Let $A$ and $A^{\prime}$ be Fuzzy sets on the universe $X$, and $B$ a Fuzzy set on $Y$. Implication, $A$ ! $B$, is defined in terms of a Fuzzy relation $R$ on the Cartesian product, $X \times Y$. The Fuzzy proposition B induced by the premise " $x$ is $A$ "'and the Fuzzy rule "if $x$ is $A$ then $y$ is $\mathrm{B}$ "is defined in the form of the Fuzzy composition. 


$$
B^{\prime}=A^{\prime} \circ R=A^{\prime} \circ(A \rightarrow B)
$$

Assume that the specific case of composition based on the Max-Min operator, then thespecial case of the above general model of Fuzzy reasoning can be defined using equation 8 as:

$$
\mu_{B^{\prime}}(y)=\bigvee_{x \in A}\left(\left(\mu_{A^{\prime}}(x) \wedge \mu_{R}(x, y)\right)=\max _{x \in A} \min \left(\mu_{A^{\prime}}(x), \mu_{R}(x, y)\right.\right.
$$

By now, in a position to build some reasoning engines. Consider the following three special cases:

1) Single Rule with Single Premise

The premise simplifies to the special case of a scalar thresholds, or $\mu_{B^{\prime}}=w \wedge \mu_{B}(y)$

2) Single Rule with Multiple Premises

The premise simplifies to the special case of the minimum of two scalar thresholds.

Thus, the compositional rule implies $C^{\prime}=\left(A^{\prime} \times B^{\prime}\right) \circ(A \times B \rightarrow C) \quad$ or $\quad \mu_{B^{\prime}}=w_{1} \wedge w_{2} \wedge \mu_{c}$.

3) Multiple Rules with Multiple Premises

The 'max' operator of equation (8) now applies, thus the area of the implication is the maximum of each minimally threshold premise.

At this point, the basis for Fuzzy reasoning with the remaining problem of establishing what the Fuzzy consequent actually means in practice. Tsukamoto Fuzzy reasoning are models based on Fuzzy Logic. These rules are easy to learn and use and can be modified according to the situation. It helps to make decisions and can be used in decision analysis. Tsukamoto Fuzzy reasoning does mapping from given input to an output using Fuzzy Logic. Tsukamoto Fuzzy reasoning has a number of rules based on if - then conditions.

In this method, the consequence of each Fuzzy rule is represented by a Fuzzy set with a monotonic membership function. The rule base has the form as: $R_{i}$ : if $\mathrm{u}$ is $A_{i}$ and $\mathrm{v}$ is $B_{i}$, then $\mathrm{w}$ is $C_{i}, \mathrm{i}=1,2, \mathrm{n}$. Where $\mu C_{i}(\mathrm{w})$ is a monotonic function. As a result, the inferred output of each rule is defined as a crisp value induced by the rules matching degree (firing strength). The overall output is taken as the weighted average of each rules output. Suppose, that the set $C_{i}$ has a monotonic membership function $\mu C_{i}(\mathrm{w})$ and that $\alpha_{i}$ is the matching degree of its rule.

For the Fuzzy set input ( $\left.A^{\prime}, B^{\prime}\right)$ is given by the equation 9:

$$
\alpha_{i}=\min \left[\max _{u}\left(\mu_{A^{\prime}}(u) \wedge \mu_{A_{i}}(u), \max _{v}\left(\mu_{B^{\prime}}(v) \wedge \mu_{B_{i}}(v)\right)\right]\right.
$$

Then the result of its rule is obtained by the equation 10 :

$$
w_{i}=\mu_{C_{i}}^{-1}\left(\alpha_{i}\right)
$$

The final result is derived from the weighted average like in the following when there are two rules. This term is given by the equation 11 :

$$
w_{0}=\frac{\alpha_{1} w_{1}+\alpha_{2} w_{2}}{\alpha_{1}+\alpha_{2}}
$$

Since each rule infers a crisp result, the Tsukamoto Fuzzy model aggregates each rules output by the weighted average method. Therefore, it avoids the time-consuming process of defuzzification.

\section{Fuzzy Logic and Dempster-Shafer Mathematical Theory of Evidence}

Fuzzy set theory proposed by Zadeh in 1965 is a kind of theoretical reasoning scheme for dealing with imperfect data. A Fuzzy set, as the name implies, is a set without a crisp boundary. That is, the transition is gradual and this smooth transition is characterized by 
membership functions. The Fuzzy inference system or Fuzzy model is a popular computing framework based on the concepts of Fuzzy set theory, Fuzzy if-then rules and Fuzzy reasoning. The basic structure of a Fuzzy inference system consists of three conceptual components which are rule base, database and reasoning mechanism. A rule base or decision matrix of the Fuzzy knowledge-base, which contains a selection of Fuzzy rules and a database, which defines the membership functions used in the Fuzzy rules composed of expert IF $<$ antecedents $>$ THEN $<$ conclusions $>$ rules. A reasoning mechanism, which performs the Fuzzy reasoning based on the rules and given facts to derive a reasonable output or conclusion. Mathematically speaking, consider $F$ to represent a Fuzzy set in the domain of discourse $U$. The Fuzzy set $F$ can be defined by the membership function from equation 12 as follows:

$$
\mu_{F}(x) \in[0,1], \forall x \in U
$$

The membership function of a Fuzzy set is a generalization of the indicator function in classical sets. In Fuzzy Logic, it represents the degree of truth as an extension of valuation. Properties of membership function are:

1) The membership function should be strictly monotonically increasing, or strictly monotonically decreasing, or strictly monotonically increasing then strictly monotonically decreasing with the increasing value of elements in the universe of discourse $X$.

2) The membership function should be continuous or piecewise continuous.

3) The membership function should be differentiable to provide smooth results.

4) The membership function should be of simple straight segments to make the process of fuzzy models easy and to high accuracy.

A new method to obtain basic probability assignment is proposed based on the similarity measure between membership function. Method to integrate Fuzzy Logic and Dempster-Shafer mathematical theory of evidence as follows:

1) Define linguistic variable and Fuzzy range. Define a variable whose values can be expressed by means of natural language terms. When defining a linguistic variable, it is also to specify minimum and maximum values.

2) Define the Fuzzy rules. The Fuzzy rules are nearly a series of if-then statements. These statements are derived by an expert to achieve optimum results.

3) Define the formula. Various other membership functions such as triangular, trapezoidal, Gaussian, and sigmoidal can be used in the formulation of membership functions.

4) Define the input. The inference schemes are based on the compositional rule of inference, and the result is derived from a set of Fuzzy rules and given inputs.

5) Calculate membership value. Calculate information contained in a fuzzy set which is described by its membership value.

6) Calculate the rule. Calculate the similarity between Fuzzy membership function to get a basic probability assignment as shown in the equation 13.

$\operatorname{Bel}(A)=\frac{\sum_{i=1}^{n} \alpha_{i}}{n(A)}$

7) For a decision problem, all the possible results from a set $m(\theta)$. Then, any proposition is a subset of $m(\Theta)$, which is called identification frame.

8) For an identification frame $m(\Theta)$ is called a function $m: 2^{\ominus} \rightarrow[0,1]\left(2^{\ominus}\right.$ is the power set of $\Theta$ ) basic probability assignment if $m$ satisfies the following conditions, as shown in the equation 1. $m(A)$ is called basic possibility assignment value, which presents the level of trust to proposition $A$.

9) For an identification frame $m(\Theta), m: 2^{\ominus} \rightarrow[0,1]$ is the basic probability assignment of $\Theta$, define function $\mathrm{Bel}$ as $\mathrm{Bel}: 2^{\ominus} \rightarrow[0 ; 1]$. $\operatorname{Bel}(\mathrm{A})$ is called belief function, as shown in the equation 3 presents the sum of all the possibilities of $A$, which is also the total evaluation of $A$. 
10) Given two belief functions $\mathrm{Bel}_{1}, \mathrm{Bel}_{2}$, which are the same identification frame, $m 1, m 2$ are corresponding basic probability assignments and their focal elements are $A 1$, $A 2, \ldots, A m$ and $B_{1}, B_{2}, \ldots, B_{n}$. The combination: $m=m 1 \oplus m 2$ is defined according to the Dempster's rule of combination as shown in the equation 14.

$$
\left(m_{1} \oplus m_{2}\right)(A)= \begin{cases}0 ; & \mathrm{A}=0 \\ \frac{\sum_{B_{i} \cap B_{j}=A} m_{1}\left(B_{i}\right) m_{2}\left(B_{j}\right)}{1-\sum_{B_{i} \cap B_{j} \neq 0} m_{1}\left(B_{i}\right) m_{2}\left(B_{j}\right)} ; & \mathrm{A} \neq 0\end{cases}
$$

\section{Conclusion}

Fuzzy reasoning does the mapping from given input to an output using Fuzzy Logic. Fuzzy reasoning models have a number of rules based on if then conditions. In fact, these rules are easy to learn and use and can be modified according to the situation. It helps to make decisions and can be used in decision analysis. Dempster-Shafer mathematical theory of evidence is one of the important tool for decision making under uncertainty. Dempster-Shafer theory makes inferences from incomplete and uncertain knowledge, provided by different independent knowledge sources. Dempster-Shafer theory provides explicit estimation of imprecision and conflict between information from different sources and can deal with any unions of hypotheses. Dempster-Shafer mathematical theory of evidence is a formal framework for plausible reasoning which provides techniques for characterizing the evidences by considering all the available evidences. Dempster-Shafer theory has been used in decision making. The knowledge is uncertain in the collection of basic events can be directly used to draw conclusions in simple cases, however, in many cases the various events associated with each other. Reasoning under uncertainty that used some of mathematical expressions, gave them a different interpretation which is each piece of evidence may support a subset containing several hypotheses. This is a generalization of the pure probabilistic framework in which every finding corresponds to a value of a variable. In this research it is Fuzzy Logic and DempsterShafer theory, which resulted in a $0 \%$ rejection. Finally, Fuzzy Logic and Dempster-Shafer mathematical theory of evidence have shown good results.

\section{References}

[1] Zadeh LA. Fuzzy Sets. Information and Control. 1965; 8: 338-353.

[2] Yen Z. Generalizing The Dempster-Shafer Theory to Fuzzy Sets. 1990; 20: 559-570.

[3] Binaghi E, Gallo I, Madella P. A Neural Model for Fuzzy Dempster-Shafer Classifiers. International Journal of Approximate Reasoning. 2000; 25: 89-121.

[4] Yager RR, Filev P. Including Probabilistic Uncertainty in Fuzzy Logic Controller Modeling Using Dempster-Shafer Theory. IEEE Transactions on Systems, Man, and Cybernetics. 1990; 25: 12211230.

[5] Dymova L, Sevastjanov P. The operations on intuitionistic fuzzy values in the framework of DempsterShafer theory. Knowledge-Based Systems. 2012; 35: 132-143.

[6] Ghasemi J, Ghaderi R, Mollaei MRK, Hojjatoleslami SA. A Novel Fuzzy Dempster-Shafer Inference System for Brain MRI Segmentation. Information Sciences. 2013; 223: 205-220.

[7] Walijewski JS, Sosnowski ZA. Genetic Tuning Fuzzy Dempster-Shafer Decision Rule. Task Quarterly. 2002; 6: 631-640.

[8] Boudraa AO, Bentabet A, Salzenstein F, Guillon L. Dempster-Shafer's Basic Probability Assignment Based on Fuzzy Membership Functions. Electronic Letters on Computer Vision and Image Analysis. 2004; 4: 1-9.

[9] Dutta P, Ali T. Fuzzy Focal Elements in Dempster-Shafer Theory of Evidence: Case study in Risk Analysis. International Journal of Computer Applications. 2011; 34: 46-53.

[10] Dempster AP. Upper and lower probabilities induced by a multivalued mapping. Ann. Math. Stat. 1967; 38: 325-339.

[11] Dempster AP. A Generalization of Bayesian inference. Journal of the Royal Statistical Society. 1968; 30: 205-247.

[12] Shafer G. A Mathematical Theory of Evidence. New Jersey: Princeton University Press. 1976. 
[13] Lyu CH, Choi MS, Li ZY, Youn HY. Reasoning with Imprecise Context Using Improved DempsterShafer Theory. In: 2010 IEEE/WIC/ACM International Conference on Web Intelligence and Intelligent Agent Technology. Toronto, Canada. 2010: 475-478..

[14] Yao S, Huang W. A New Approach for Multi-attribute Decision Making Problem with Incomplete Information Based on Dempster-Shafer Theory. In: International Conference on Management Science and Industrial Engineering (MSIE). Harbin. 2011: 620-623.

[15] Yu X, Li M, Ping Z. Dempster-Shafer Theory as an Applied Approach to Scenario Forecasting Based on Imprecise Probability. In: IEEE 12th International Conference on Computer and Information Technology. Chengdu, China. 2012: 975-980.

[16] Uphoff C, Gritschneder DM, Schlichtmann U. Application of Dempster-Shafer Theory to Task Mapping under Epistemic Uncertainty. In: IEEE International Systems Conference (SysCon). Orlando, FL, USA. 2013: 536-541.

TELKOMNIKA Vol. 16, No. 3, December 2015 : $583-590$ 